\title{
The application of micro area analysis of Al-Cu junction by wavelength-dispersive EPMA equipped with a FE electron gun.
}

Takashi Kimura, Toshiya Ogiwara, Sei Fukushima and Shigeo Tanuma

Materials Analysis Station, National Institute for Materials Science

1-2-1 Sengen, Tsukuba, Ibaraki 305-0047, JAPAN

We have successfully developed a new-type wavelength-dispersive electron probe micro analyser (FE-WD-EPMA) equipped with a Shottoky-type FE emitter, five wavelengh-dispersive spectrometers and one energy-dispersive spectrometer[1]. In recent year, Auger electron spectrometer (AES) and SEM-EDS are effectively utilized with the FE electron gun for a submicron meter region analysis. However, mounting the FE electron gun in WDS-EPMA system was very hard, because the high vacuum system is required for FE electron gun. Why the argon (Ar) and methane $\left(\mathrm{CH}_{4}\right)$ gas, which are used for the proportional X-ray detector, may leak out in to the vacuum. Then, we have developed an evacuation system of electron gun as follows; Evacuation system of electron gun and sample chamber are divided by an intermediate vacuum system. The intermediate and sample chambers are evacuated by turbo molecular pump and rotary pump respectively. As the result of these innovations, two steps ion pumps evacuate the electron gun to high vacuum of $10^{-8} \mathrm{~Pa}$. In addition, long-term stability of electron probe current is realized which is so good for employing schottky type field emission electron gun.

The FE-WD-EPMA produces a small electron probe diameter with large probe current, particularly at low accelerating voltages. Due to this improvement, we have achieved higher spatial resolution, enhanced detectability in the elemental distribution, and high-speed elemental mapping. In this study, the results of analysis by FE-WD-EPMA of various intermatallic compounds, which grew in the interface of $\mathrm{Al}$ and $\mathrm{Cu}$ junction, were described.

Fig. 1 shows the comparison of the back-scattered electron images (BEI) of Al-Cu junction taken by a conventional tungsten-hairpin (W) gun and by FE Gun. The Analysis condition was also equal to $\mathrm{W}$-gun and FE-gun, as $10 \mathrm{kV}-50 \mathrm{nA}-50 \mathrm{msec} /$ pixel and total measurement pixels were $1024 \times 256$. The spatial resolution of the image obtained by FE-Gun is higher than conventional W-gun. And the total acquisition time of this data was less than the half of that by W-gun.

Fig.2 shows identification result of the $\mathrm{Al}-\mathrm{Cu}$ intermetallic compound by the scatter diagram method. In the scatter diagram with W-gun (a), the existence of $\theta$ and $\eta_{2}$ layer could be confirmed clearly. In addition, the $\theta, \eta_{2}, \zeta_{2}, \delta, \alpha_{2}$ and $\gamma_{1}$ layers were observed in the scatter diagram with FE-gun (b). 
Reference

[1] T. Kimura, K. Nishida, S. Tanuma and H. Yamada, J. Surface of Analysis. 10, 3(2003) 203-211

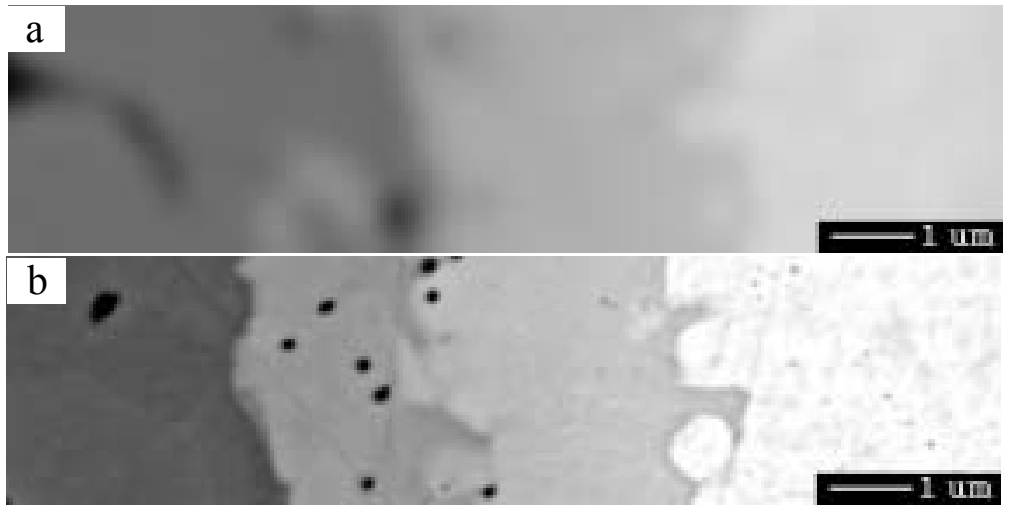

Fig.1 Comparison of the back-scattered electron images (BEI) of Al-Cu junction.

(a) W-Gun, (b) FE Gun
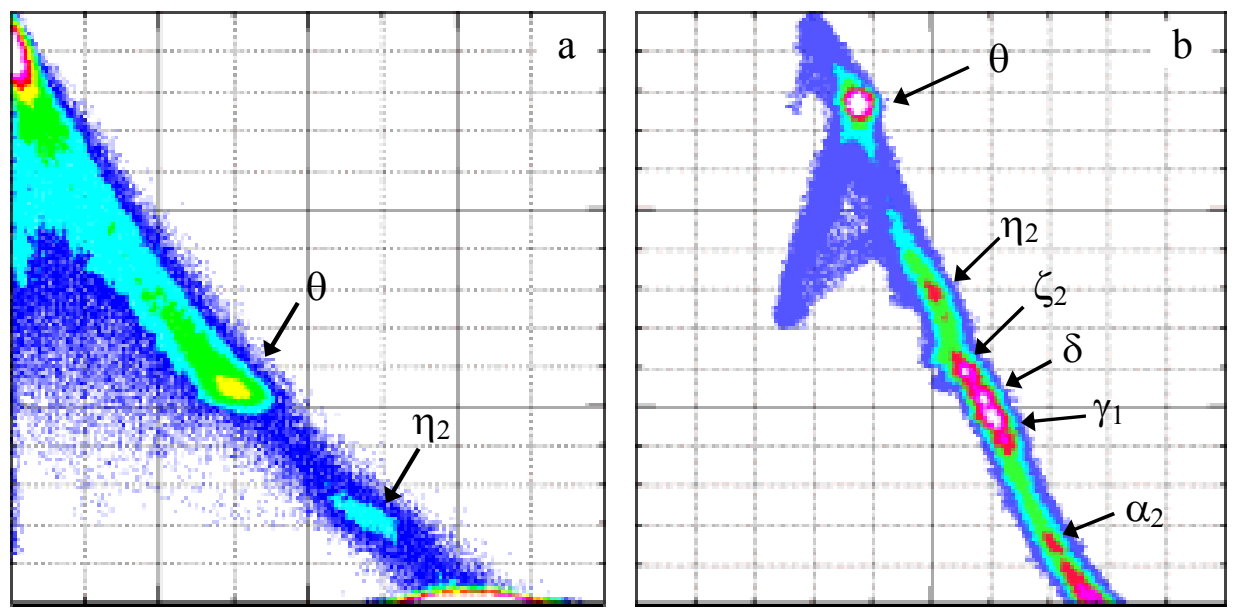

Fig.2 Identification result of the Al-Cu intermetallic compounds by the scatter diagram method.

(a) W-Gun, (b) FE Gun 